\title{
Aftereffect of inspection of a perspectival stimulus for slant depth: A new normalization effect*
}

\author{
WILLIAM EPSTEIN and CYNTHIA L. MORGAN-PAAP \\ University of Wisconsin, Madison, Wisconsin 53706
}

\begin{abstract}
The Ss made judgments of the frontoparallel plane monocularly and binocularly by adjusting the position of a rotatable thin luminous line viewed in darkness. The judgments were made before and after a 10-min period of monocular inspection of a luminous outline frontoparallel trapezoid which was the projection of a rectangle rotated in depth. Verbal reports indicated normalization of perspectival slant depth, i.e., the inspection figure appeared less rotated, and the line settings exhibited an aftereffect of normalization. These findings may contribute toward an account of certain previously unexplained observations reported in an earlier study by Epstein and Morgan-Paap (1974) dealing with adaptation to uniocular image magnification.
\end{abstract}

Under certain viewing conditions, an objectively frontoparallel form which duplicates the frontoparallel projection of a rotated modal form, e.g., a rectangle or a circle, will appear to be rotated in depth. For example, a frontoparallel luminous trapezoid presented in otherwise dark surroundings will appear to be rotated in depth by an amount that conforms to the degree of rotation that would have to be imposed on a rectangle to produce a projective shape equivalent to the frontoparallel trapezoid (Epstein \& Morgan-Paap, 1974, Experiment I). This is an instance of the perspectival determination of perceived slant depth. The present experiment was designed to learn whether continuous inspection of a perspectival stimulus for depth results in normalization of perspectival depth. During the course of monocular observation of a frontoparallel perspectival stimulus for slant depth, will the target appear to depart from its initial, apparently rotated, position to a position closer to frontoparallel (normalization), and will this shift influence subsequent depth judgments (aftereffect)?

The following general plan was adopted: First, $S$ provided a set of judgments of the apparent frontoparallel plane by rotating a thin luminous line into the frontoparallel plane. Then for $10 \mathrm{~min}, \mathrm{~S}$ engaged in monocular inspection of a frontoparallel, apparently slanted, trapezoid. The experimental session concluded with a second set of frontoparallel line settings. Each $\mathrm{S}$ participated in two sessions on different days, one requiring monocular frontoparallel settings, the other requiring binocular settings.

*This investigation was supported by Research Grant MH16390 awarded to the first author by the National Institute of Mental Health. The authors are indebted to Kenneth Paap and Wayne Shebilske for assistance in preparation of the manuscript.

\section{METHOD}

\section{Apparatus}

The inspection figures were presented in a viewing box so that the figure appeared to be luminous in a totally dark surrounding. (For details, see Epstein \& Morgan-Paap, 1974, Experiment II). The figures was located at a distance of $154.48 \mathrm{~cm}$ directly in front of $S$. $S$ viewed the target when positioned in a chinrest and head-clamp arrangement. At a distance of $101 \mathrm{~cm}$ to the left of the chinrest and head clamp, a second chinrest and head-restraining arrangement was set up on the same level as the first chinrest. At a distance of $154.48 \mathrm{~cm}$ from $\mathrm{S}$, a rotatable electroluminescent line, $29 \mathrm{~cm}$ long and $.5 \mathrm{~cm}$ wide, was located. The height of the line was at S's eye level. The line could be rotated in the horizontal plane on a vertical axis about a pivot point which remained fixed directly in front of $S$. The position of the line could be read to an accuracy of $.5 \mathrm{deg}$ of rotation.

\section{Stimuli}

The inspection figures were photographic negatives of a $20.4 \mathrm{x}$ $10.2 \times 1.9 \mathrm{~cm}$ wide rectangle, photographed at slants of $\pm 20, \pm 40$, and $\pm 60 \mathrm{deg}$ (right side forward, left side forward). The negatives were placed in slots in front of frosted glass and were backilluminated. They appeared to $S$ to be luminous white against a totally black background.

\section{Design}

Three independent groups of $12 \mathrm{Ss}$ were tested, each assigned to a different inspection figure. Within each group, 6 Ss viewed the projection with the right side apparently forward and 6 Ss viewed the projection with the left side apparently slanted forward. Each $S$ saw only one inspection figure. When viewing the inspection figure. $S$ was restricted to monocular vision.

Each $S$ was tested twice, once with binocular viewing of the rotatable line and once with monocular viewing. Within each group, half of the Ss had the monocular test condition on the first day and the binocular test condition $48 \mathrm{~h}$ later, while the other Ss had the two test conditions in reverse order. All monocular viewing conditions used the right eye. The left eye was covered by an eye patch.

\section{Procedure}

First, Ss made six judgments of the frontoparallel position of the electroluminescent line by instructing $E$ to adjust the position of the line until it appeared parallel with the forehead. The $S$ was seated at the chinrest and viewed the electroluminescent line in totally dark surroundings. Under the monocular test condition, $\mathrm{S}$ was 
encouraged to rotate his head on the chinrest, with no restraint imposed except that $S$ was required to keep on the chinrest. Under the binocular test condition, S's head was held stationary by a head-restraining arrangement. Head movements were explicitly discouraged.' Three of the six prenormalization settings started with the left end of the line $25 \mathrm{deg}$ back and three with the right end $25 \mathrm{deg}$ back. The two starting positions were ordered randomly.

Following the prenormalization.test, Ss moved to the inspection viewing position and the following instructions were provided: "This stage of the experiment is concerned with another form of depth judgment. We wish to learn whether the depth at which objects appear varies over a $10-\mathrm{min}$ period of continuous observation. When you look into this box, you will see a luminous four-sided figure in an otherwise dark background. At infrequent and irregular intervals, a faint light will be flashed inside the figure. The light will appear in one of the four corners. The corners at which the light will appear have been selected randomly, so it will not be possible for you to predict where the signal will appear. Each time a light flashes. I want you to answer two questions about the corner in which the light appeared. (1) Does the corner, that is, the corner where the light appeared, appear nearer, farther, or equal in distance from you as the corner to the right or left? (2) Does the corner appear nearer, farther, or equal in distance from you as the corner below or above it?" An example was presented and, when S understood the procedure, the 10-min inspection period was initiated. The light flashes were brief and appeared at random times at intervals ranging from 30 to $90 \mathrm{sec}$ in randomly selected corners.

At the conclusion of the inspection period, $S$ closed her eyes while she returned to the test viewing position. The time required to move from the inspection position to the test position and commence the first posttest judgment never exceeded $30 \mathrm{sec}$. All Ss made two postnormalization judgments of the frontoparallel position of the electroluminescent line.

Prior to initiating the main experiment, a control study was conducted to assess the effects on frontoparallel settings of the between-eye differences in dark adaptation (von Békésy. 1970) that might occur in the main experiment as a result of the monocular occlusion during the 10 -min inspection period. The control Ss were tested with the same experimental procedure described above, but using a 0 -deg (rectangle) inspection figure instead of an apparently rotated figure. As in the main study, half of the Ss were tested monocularly the first day and binocularly on the second day, while the other $S$ s had the two test conditions in reverse order. If differential dark adaptation affects the frontoparallel setting, then an effect should appear with the 0-deg inspection figure and this effect would have to be taken into account in assessing depth normalization.

\section{Subjects}

Thirty-six undergraduates from the University of Wisconsin served as Ss in the main experiment. Twelve Ss were assigned to each of the three inspection figures. Six other undergraduates served as $S s$ in the control experiment. None of the Ss wore framed corrective glasses, but several were regular users of contact lenses and these Ss continued to wear the contact lenses throughout the experiment.

\section{RESULTS}

\section{Control Experiment}

Preinspection Judgments. The mean preinspection frontoparallel judgments, based on the last two of the six settings, show that Ss were highly accurate. The mean for the $\mathrm{Ss}$ for the monocular test was $180.92 \mathrm{deg}$; the mean for the binocular test was $180.67 \mathrm{deg}$. A reading of $180 \mathrm{deg}$ signified that the line was perpendicular to $S$ 's line of sight, that is, in the frontoparallel position; a reading of over $180 \mathrm{deg}$ indicated that the left side was rotated back; a reading of under $180 \mathrm{deg}$ indicated that the right side was rotated back. A 1 between-Ss (order of test condition), 1 within-Ss (test condition) ANOVA of the preinspection settings showed no significant main effects or interactions.

Posinspection Judgments. The mean postinspection frontoparallel judgments were $180.75 \mathrm{deg}$ for both binocular and monocular test conditions. The monocular condition exhibited a shift of $-0.17 \mathrm{deg}$ and the binocular condition a shift of $0.08 \mathrm{deg}$, both clearly insignificant by analysis of variance. Under the conditions that prevail in our experiments, differential dark adaptation does not affect the apparent frontoparallel. Also, these data serve to allay concern that uncontrolled test-retest effects may produce shifts independently of the influence of the inspection figure.

\section{Main Experiment}

Preinspection Judgments. The results are summarized in Table 1. The mean preinspection frontoparallel judgments, based on the last two of the six settings, show that Ss were highly accurate. A 3 between-Ss (direction of perspectival slant, degree of slant, order of test conditions), 1 within-Ss (test condition) ANOVA of the preinspection settings showed no significant main effects or interactions. Therefore, we may assume that all groups were alike prior to commencing the inspection period.

Postinspection Judgments. Normalization is evident if after the inspection period $S$ judged the line to be frontoparallel when the line was slanted in the direction of the slant of the inspection figure. Those who inspected a figure representing a left-side away slant, should set the line with the left side farther

Table 1

Mean Preinspection Settings of Frontalparallel and Mean Pre-Post Inspection Shift

\begin{tabular}{|c|c|c|c|c|c|c|}
\hline & \multicolumn{6}{|c|}{ Conditions of Inspection } \\
\hline & \multicolumn{2}{|c|}{$20 \mathrm{Deg}$} & \multicolumn{2}{|c|}{$40 \mathrm{Deg}$} & \multicolumn{2}{|c|}{$60 \mathrm{Deg}$} \\
\hline & Monoc & Binoc & Monoc & Binoc & Monoc & Binoc \\
\hline $\begin{array}{l}\text { Preinspection Mean } \\
\text { Preinspection SD }\end{array}$ & $\begin{array}{r}180.46 \\
1.46\end{array}$ & $\begin{array}{r}180.42 \\
1.52\end{array}$ & $\begin{array}{r}180.17 \\
1.62\end{array}$ & $\begin{array}{r}180.21 \\
1.01\end{array}$ & $\begin{array}{r}180.42 \\
1.25\end{array}$ & $\begin{array}{r}180.00 \\
1.15\end{array}$ \\
\hline $\begin{array}{l}\text { Shift Mean (Deg) } \\
\text { Shift SD }\end{array}$ & $\begin{array}{r}17.58 \\
4.47\end{array}$ & $\begin{array}{l}9.17 \\
3.22\end{array}$ & $\begin{array}{r}16.04 \\
3.98\end{array}$ & $\begin{array}{l}9.62 \\
2.53\end{array}$ & $\begin{array}{r}18.75 \\
4.20\end{array}$ & $\begin{array}{l}9.29 \\
2.74\end{array}$ \\
\hline
\end{tabular}


away, while those who inspected a figure representing a right-side away slant should set the line with the right side farther away.

All Ss showed shifts in the direction of normalization, with mean shifts ranging from 9.17 to $18.75 \mathrm{deg}$. The degree of shift depended only on test condition. The shift was greater for monocular condition (17.46 deg) than for the binocular condition (9.36 deg). The degree of slant depth of the inspection figure had no significant effect. A 3 between-Ss 1 within-Ss ANOVA was performed on the shift data. The main effect of test condition was significant, $F(1,24)=120.70, p<.001)$, while all other main effects and interactions were insignificant.

\section{DISCUSSION}

The aftereffect of the inspection period strongly indicates normalization of perspectival slant depth. Additional support for this interpretation was provided by the spontaneous remarks supplied by the Ss during the inspection period. Of the $36 \mathrm{Ss}, 17$ reported that for a portion of the inspection period the figure assumed a frontoparallel position and $11 \mathrm{Ss}$ reported that the figure appeared less rotated.

The fact that the shift was almost twice as great on the monocular test than on the binocular test suggests that the shift is specific to the perspectival determinants of depth. We may assume that the principal cue for depth-slant under the monocular condition of testing was the perspectival cue. Therefore, in setting the line under this condition, $S$ would be satisfied with a setting which approximated the perspective transformation represented by the inspection figure. When binocular cues were present as in the binocular test condition, the result was a compromise between the demands of normalized perspective and undistorted binocular input. On the basis of our previous work (Epstein \& Morgan-Paap, 1974, Experiment I), showing complete dominance of perspectival slant over stereoscopic slant, we would expect that the difference between the monocular and bincoular test would not have been present if the test stimulus had been a more obvious perspective stimulus, e.g., a rectangle of dimensions similar to those of the inspection figure.

The absence of a significant effect associated with the degree of inspection slant depth was not unexpected. Similar evidence of independence of depth normalization has been reported for the depth associated with optical texture (Bergman \& Gibson, 1959; Wenderoth, 1970) and for depth associated with disparity (Howard \& Templeton, 1964; Blakemore \& Julesz, 1971). It is not clear why this finding is consistently obtained, especially since where adaptation to experimentally produced optical transformations is concerned, e.g., as in prism adaptation, the magnitude of the shift is roughly proportional to the magnitude of the transformation.
Studies of lesser degrees of perspectival slant may be useful in examining this question.

Normalization effects are usually small. This is borne out by a review of studies of tilt, curvature, and depth normalization. The size of the effect in the present experiment certainly is atypical. We believe the explanation resides in the nature of the stimuli. Under the viewing conditions, the luminous trapezoid is an equivocal stimulas that allows different perceptual resolutions: the inspection figure may appear as a rectangle slanted in depth, a frontoparallel trapezoid or any other shape-slant combination. For a variety of reasons, the first alternative is overwhelmingly favored at the outset, but the availability of the aforementioned alternatives probably does not go unnoticed. The availability of a perceptual resolution localizing the figure in the frontal plane that is as acceptable as a model of the stimulus as the slanted-in-depth rectangle creates a favorable context for the normalization effect.

The special characteristics of the test stimulus may also have contributed to the magnitude of the change. The dimensions of the rotatable rod and the viewing distance were such that in order to produce a noticeable perspective transformation the rod had to be significantly displaced from the objective frontal plane. This necessity will be appreciated by noting that the maximum possible difference between the visual angles subtended by the near and far ends of the lines was $1 \mathrm{~min}, 48 \mathrm{sec}$ of arc. The need to introduce a large displacement of the rod in order to produce a perspective stimulus approximating the normalized perspective of the inspection figure would lead to an exaggerated response and an overestimation of the normalization effect. The obtained magnitude of the normalization may be an artifact of measurement.

Although the demonstration of perspective normalization is new, the effect is similar to a more generally established phenomenon called the "equidistance tendency" by Gogel (1965). Under a variety of conditions, but most readily under reduced viewing conditions and for objects that are adjacent. objects separated in depth will appear equidistant. In our case, the various parts of the inspection figure tended to appear equidistant or all in the frontoparallel plane. This is not to assert that the equidistance tendency accounts for our normalization effect since there currently is no general explanation of the tendency, and the tendency for objects to appear equidistant may have different explanations in different situations. For the present, we only wish to note the similarity between perspectival depth normalization and the equidistance tendency.

The present experiment demonstrated normalization in monocular viewing. If we may assume that normalization also occurs in binocular viewing, we may be able to formulate an account of an otherwise perplexing finding reported in an earlier experiment 
by Epstein and Morgan-Paap (1974, Experiment 11). In that study, we showed that a period of exposure to informational discrepancy involving stereoscopic slant depth and perspectival slant depth leads to a shift in depth judgments based on retinal disparity. For example, in one condition, $S$ wore a meridional size lens (MSL) in front of one eye which induced a horizontal binocular disparity equivalent to that produced by rotating a plane surface $60 \mathrm{deg}$ in depth. While wearing the lens, $S$ inspected a luminous frontoparallel trapezoid representing a rectangle rotated $20 \mathrm{deg}$ in depth. Following a 10 -min exposure period, Ss exhibited an aftereffect: the apparent frontoparallel plane shifted in the direction of the MSL-induced rotation. The aftereffect was explained in the following manner: When perspectival and stereoscopic slant are discrepant, the former dominates. The results of prolonged pairing of a dominant and subordinate cue is that the latter is recalibrated to become consistent with the dominant cue. The recalibration persists for a time after the dominant cue has been removed. It is this persisting recalibration which appears as an aftereffect. This account, recalibration by pairing, has been applied with a fair measure of success to a variety of findings (Epstein, 1971, 1972; Epstein \& Morgan, 1970).

Although the results of the study by Epstein and Morgan-Paap (1974) were largely consistent with the foregoing account, there was one finding which did not fit: a shift was observed when perspectival slant and stereoscopic slant matched. Since there was no discrepancy in this situation, there is no obvious way to explain the shift in stereoscopic depth in terms of recalibration by pairing. One possibility is that stereoscopic depth normalization is responsible for the shift. Another alternative is suggested by the present findings. If, during the course of inspection, normalization of perspectival slant depth occurred, this could have introduced a functional discrepancy between perspective depth and disparity depth in the condition where the two were nominally marched. This discrepancy may have been the basis for recalibration of disparity in this condition.

\section{REFERENCES}

Bergman, R., \& Gibson, J. J. The negative aftereffect of a surface slanted in the third dimension. American Journal of Psychology, 1959, 72, 364-374.

Blakemore, C.. \& Julesz, B. Stereoscopic depth aftereffect produced without monocular cues. Science, 1971, 171, 286-288.

Epstein, W. Adaptation to uniocular image magnification after varying preadaptation activities. American Journal of Psychology, 1971, 84, 66-74.

EPsTEIN, W. Retention of adaptation to uniocular image magnification: Effect of interpolated activity. Journal of Experimental Psychology, 1972, 92, 319-324.

Epstein, W., \& Morgan, C. L. Adaptation to uniocular image magnification: Modification of the disparity-depth relationship. Anerican Journal of Psychology. 1970, 83, 322-329.

Epstein, W., \& Morgan-PaAp, C. L. The effect of level of depthprocessing and degree of informational discrepancy on adaptation to uniocular image magnification. Journal of Experimental Psychology, 1974, 102, 585-594.

GoGEl, W. C. Equidistance tendency and its consequences. Psychological Bulletin, 1965, 64, 153-163.

HowARD, I., \& TEMPLETon, W. The effect of steady fixation on the judgment of relative depth. Quarterly Journal of Experimental Psychology, 1964, 16, 193-203.

VON BÉKÉSY, G.Apparent image rotation in stereoscopic vision: The unbalance of the pupils. Perception \& Psychophysics, 1970, 8, 343-347.

Wenderoth. P. M. A visual spatial aftereffect of surface slant. American Journal of Psychology, 1970, 83, 576-590.

\section{NOTE}

1. Our objective was to insure that accuracy (variability) on the prenormalization test would be comparable under the conditions of monocular and binocular viewing. Preliminary observations showed that judgments under conditions of monocular, stationary observations were more variable than binocular judgments. For this reason we allowed head movements in the monocular condition. The introduction of parallax had the desired effect.

(Received for publication February 20, 1974; revision received May 24, 1974.) 